\title{
Eating behaviours, genetic influence and dietary intake of Irish twins
}

\author{
R. Barron, K. Bermingham, L. Brennan, E. Gibney, M. Ryan and A. O’ Sullivan \\ Institute of Food and Health, University College Dublin, Dublin, Republic of Ireland
}

A growing body of evidence suggests that eating behaviours are to some extent influenced by genes ${ }^{(1)}$. Twin studies are a useful resource that can evaluate the heritability (proportion of the variation due to genetic factors) of a trait. The Dutch Eating Behaviour Questionnaire (DEBQ) is frequently used to measure three domains of eating behaviour; emotional eating (overeating in the presence of negative emotions), external eating (overeating in response to external stimuli) and dietary restraint (the tendency to consciously restrict food) ${ }^{(2)}$. The intuitive eating concept (assessed through the Intuitive Eating Scale (IES)) is based on eating in response to physiological cues ${ }^{(3)}$. This study aims to determine the presence of genetic effects on components of eating behaviour.

Data was obtained from monozygotic $(M Z)$ twins $(n=88)$ and dizygotic $(D Z)$ twins $(n=38)$. Dietary data were collected using $24 \mathrm{hr}$ recalls on 5 separate non consecutive days and assessed using WISP version 3.0 (Tinuviel Software). Eating behaviour was assessed through DEBQ and IES. High, medium and low scorers of eating behaviours were classified based on tertiles. Partial correlations controlling for age and gender were compared between MZ and DZ twins. Ethics was obtained from the UCD Human Research Ethics Committee.

Significant MZ correlations were observed for all eating behaviour components except DEBQ Restrained (Table 1). MZ correlations were significantly higher than DZ correlations for DEBQ Emotional, indicating the presence of genetic effects (Table 1). A significant difference in energy intake (kcal) was also observed between high and low DEBQ Restrained scorers, with low scorers reporting higher energy intakes (Figure 1). These findings suggest that eating behaviours may be influenced by genetic makeup. However more work is required to determine the impact of genetics and/or environment using more advanced modelling techniques ${ }^{(1)}$.

Table 1.

\begin{tabular}{llrl}
\hline & $\mathrm{MZ}$ & $\mathrm{DZ}$ & \\
\cline { 2 - 4 } & $\mathrm{r}=$ & $\mathrm{r}=$ & $p<$ \\
\hline DEBQ Emotional & 0.506 & 0.014 & $0.041^{*}$ \\
DEBQ External & 0.667 & 0.166 & 0.119 \\
DEBQ Restrained & 0.254 & -0.139 & 0.139 \\
IES Total Score & 0.721 & 0.370 & 0.192 \\
\hline
\end{tabular}

Partial correlations controlling for age and gender

* Significant at $p<0.05$

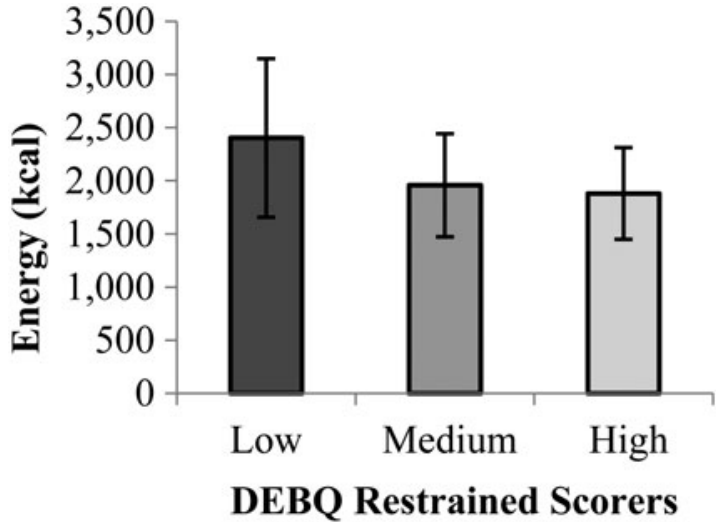

Fig. 1.

1. Sung J, Lee K, Song YM et al. (2010) Obesity (Silver Spring) 18, 1000-5

2. Van Strien T, Frijters JE, Bergers G, et al. (1986) Int J Eat Disord 5, 295-315

3. Hawks S, Merrill RM, Madanat HN. (2004) J Health Educ 35:90-9. 